\title{
High Rate Squeezing using LL Band Coding in Wavelet Domain
}

\author{
M. Santhosh
}

\begin{abstract}
Wavelet based image compression standards not only inspired signal and image processing community but also the research community of many research and application fields towards the wavelet theory. All wavelet based schemes follow the standard sequence of steps. They are transformation and the processing task at one end followed by the inverse of processing task and inverse transform at another end. Wavelet based compression was done in a quite different manner from its inception. The early techniques include Embedded Zerotree Wavelet (EZW) coding and Set Partitioning in Hierarchical Trees (SPIHT) coding. Although, SPIHT is an extension of $E Z W$, both follow more or less similar process in coding and decoding. These schemes code the significant and insignificant coefficients using symbols or maintaining a list of indices of the coefficients. The decision on significant or insignificant will be taken by comparing with a threshold which will be updated in each iteration. In both the schemes, if a coefficient is identified as an insignificant one, then the bits incurred in conveying this coefficient is less and in many cases very less. One can imagine that if a coefficient is made to be an insignificant then the number of bits required will be less. This issue was taken up in this paper and bits of selected regions is chosen and a significant improvement is compression ratio is observed at a little cost of quality.
\end{abstract}

Keywords: EZW, significance map, SPIHT, wavelet based compression

\section{INTRODUCTION}

Wavelet transform is widely used in many applications of image processing and it is extensively used in image compression wherein, the wavelet based image compression standard, JPEG2000 [1] is one of the popular compression technique that has outperformed JPEG [2], the traditional coding based on DCT. The compression ratio entirely depends on the choice of wavelet when wavelet transform is used. Hence, standard wavelets are used which are not adaptive to the given image but have an impact on photographic images. It is a well-known fact that similar images may not have similar or same statistical measures like that of photographic images viz., medical images, documents that are scanned, satellite imagery, fingerprint images etc. Standard wavelets, when used in image coders for compression do not yield much compression as they are not fit for such images. Besides, large database will be maintained for storing similar non photographic images, leading to the necessity for the development of adaptive wavelet for compression.

Current day technology has made individuals to have multimedia enabled data services which require immense data transfer on the fly as well. Hence, there is a need for rapid transmission of data based on demand. Such a fast transmission of data is possible only when the bandwidth is sufficient. Hence, it can be concluded that in terms of image processing, image storage and transmission plays a vital role in such transmission. It is already notified that there will be drastic improvement in data rates as the users switch from Long Term Evolution (LTE) to Large Scale Convergence (LSC). A solution need to devise for such increased data rates. Hence, reduction in the volume of the data that is being intended for transfer or use of compression techniques are the two solutions that mitigate the problem. JPEG [3-4], JPEG2000 [5-6], and MPEG [7] are recently developed techniques for compression that has achieved better compression having no compromise on the quality of the image. But, the energy that is consumed during compression and transmission, in particular, in wireless transmission is not given appropriate weight. Hence, this paper exploits this problem and proposes an efficient image compression technique by the use of selective wavelet domain. Further, it is proposed to reduce the computational overhead in processing as well that will occur during the computation of certain high-pass coefficients.

Wavelets are the one of the best suited application in image analysis as wavelets provide frequency as well as spatial information of the images. Such a spatial information will be available in different scales for different sizes of the images [8]. The fundamental limitation of any transform is that there exists no relation between the input signal and the basis function used in the transformation including wavelet transform [9]. This is because the input signal details may be completely different from the features available in basis function [10-14]. Though this limitation has less effect in the case of wavelet as the basis function in wavelet is not fixed and also it is generally translated and dilated. Apart from that, the decomposition bands generated in wavelet transform has different effect from each other. In image analysis, the wavelet transform generates four decomposition bands at level 1, out of which one has less influence on reconstruction. Hence, in this paper a scheme is proposed to selectively choose the decomposition bands in reconstruction. This selective decomposition bands will have a significant effect, in particular, in image compression. 
HIGH RATE SQUEEZING USING LL BAND CODING IN WAVELET DOMAIN

\section{WAVELET BASED IMAGE COMPRESSION SCHEMES}

One of the most successful applications of wavelet transforms is transform-based compression. The coding scheme first, removes the redundancy of the wavelet domain, then quantizes and finally some sort of entropy coding will be applied. As the wavelet transform possess superior energy compaction feature, the wavelet based schemes produces best subjective as well as objective performance. Both orthogonal and bi-orthogonal wavelets are used for image compression. Many high-quality algorithms use some sort of transform coding. One such popular scheme is JPEG. JPEG in its initial version makes use of discrete cosine transform (DCT). In this scheme, input image is divided into $8 \times 8$ blocks. Each block is transformed using 8-bit DCT.

The transformed coefficients are organized as 64 subbands. Then these are scalar quantized and consequently coded with Huffman coding. The JPEG standard results in compression of up to 10:1. But, at medium and high compression rates, an effect called blocking artifact degrades the quality of reconstructed image. This is because the fundamental principle of JPEG is to divide the input image into blocks of specific size. When these blocks, after transformation, are extremely coded, the boundaries appears resulting in annoyances in visualization.

\section{a. EZW Coding}

EZW is the one of the early algorithms to show the power of wavelet-based image compression. For that matter, the proposal of EZW by Shapiro in 1993 [11], created a buzz not only in the image processing community, but also in many Engineering and Sciences fields, where many algorithms were proposed using wavelets. Embedded coding is a process of coding the transformed coefficients by using which progressive transmission is made possible. EZW coding contains two passes in the coding. They are Significance pass and refinement pass.

As an example, consider the wavelet decomposition given below.

\begin{tabular}{|c|c|cc|}
\hline 53 & 13 & 27 & 21 \\
\cline { 1 - 2 }-15 & 15 & 13 & 9 \\
\hline 9 & -9 & 9 & -7 \\
5 & -5 & -5 & 0 \\
\hline
\end{tabular}

The threshold will be calculated using the following equation.

$$
T=2^{\log _{2}^{C_{M a x}}}
$$

where $\mathrm{C}_{\text {Max }}$ is the maximum coefficient value in the wavelet decomposition. The threshold will be halved in further phases. It is to note that the terms 'phase' and 'pass' are used to refer the same in the paper. In the first phase, the maximum coefficient is 53 . Hence, the threshold becomes 32. Now the coefficients in the wavelet decomposition need to be traversed in the order shown in Fig. 1.

\begin{tabular}{|c|c|c|c|c|c|c|c|}
\hline 1 & 2 & 5 & 8 & 17 & 24 & 25 & 32 \\
\hline 3 & 4 & 6 & 7 & 18 & 23 & 26 & 31 \\
\hline 9 & 10 & 13 & 14 & 19 & 22 & 27 & 30 \\
\hline 12 & 11 & 15 & 16 & 20 & 21 & 28 & 29 \\
\hline 33 & 34 & 35 & 36 & 49 & 50 & 54 & 55 \\
\hline 40 & 39 & 38 & 37 & 51 & 53 & 56 & 61 \\
\hline 41 & 42 & 43 & 44 & 52 & 57 & 60 & 62 \\
\hline 48 & 47 & 46 & 45 & 58 & 59 & 63 & 64 \\
\hline
\end{tabular}

Fig. 1 Scanning order of wavelet domain coefficients for 8x8 decomposition: zigzag through all-low pass subband and diagonal subbands, column scan through vertical subbands, row scan through horizontal subbands.

The first element in the sequence is 53. This element will be compared with the threshold which is 32 . The coefficient 53 is higher than the threshold and hence it is significant. The comparison will always be made with respect to the magnitude. If the magnitude is greater than the threshold, then that coefficient may be coded as either a significant positive ' $p$ ' or a significant negative ' $n$ '. The next coefficient in the traversal is 13 which is lesser than the threshold, in addition, all the descendants 27, 21, 13 and 9 are also smaller than threshold forming a zerotree. Hence the coefficient 13 is coded as a zerotree ' $t$ '. Next coefficients -15 and 15 are also smaller than the threshold and also the descendants of these are also smaller than the threshold. Hence two more zerotrees will be formed. The sequence of symbols generated as of now is ' $p$ - $t-t-t$ '. The coefficients treated as significant need refinement pass. Hence there is only one coefficient which need refinement, i.e. 53. Hence this coefficient will be queued in a list, i.e. $\operatorname{LIST}_{\text {Sub }}=\{53\}$. Reconstructed value will calculated using the expression ' $1.5 \times \mathrm{x}$ ', $\mathrm{T}$ is the threshold at the current pass. Hence, the reconstructed value becomes 48 . The wavelet domain now becomes,

\begin{tabular}{|c|l|ll|}
\hline 48 & 0 & 0 & 0 \\
0 & 0 & 0 & 0 \\
\hline 0 & 0 & 0 & 0 \\
0 & 0 & 0 & 0 \\
\hline
\end{tabular}

In the subordinate pass, a correction term will be evaluated and reconstructed values will be corrected accordingly. The magnitude of correction term is always T/4 and the sign is the sign of the subtraction of reconstructed value from original coefficient value. In the current case '53-48', which is positive, hence the reconstruction results in $48+(\mathrm{T} / 4)=48+8=56$. When a fixed length coding is used, 2-bits will be used to each symbol generated and for the sign of correction term one bit, a total of 9 bits will be used to get the following wavelet domain.

\begin{tabular}{|c|c|cc|}
\hline 56 & 0 & 0 & 0 \\
0 & 0 & 0 & 0 \\
\hline 0 & 0 & 0 & 0 \\
0 & 0 & 0 & 0 \\
\hline
\end{tabular}


With is this, the phase is complete. In the next phase, the threshold value becomes 16 . It is to note that the coefficient ' 53 ' is already processed and it will be considered only in reconstruction in every phase. The first element in the current traversal is ' 13 ', which is lesser than the threshold ' 16 '. But, some of the descendants of ' 13 ' are greater than the threshold. Hence, ' 13 ' is treated as isolated zero ' $z$ '. The next two coefficients to be traversed are ' -15 ' and ' 15 '. Both are lesser than the threshold and their descendants are also smaller than the threshold, hence ' -15 ' and ' 15 ' become zerotree roots, and will be coded as ' $t-t$ '. Now, four coefficients are remained in the current dominant pass. They are ' 27 ', ' 21 ', 13' and ' 9 '. The first two are significant positives and the remaining are zerotree roots. Hence the complete sequence of symbols in this dominant pass becomes, ' $\mathrm{z}$ - $\mathrm{t}-\mathrm{t}-\mathrm{p}$ - $\mathrm{p}$ - $\mathrm{t}-\mathrm{t}$ '. The two new significant coefficients will be added to the subordinate list, hence $\operatorname{LIST}_{\text {Sub }}=\{53,27,21\}$. The reconstruction value is $1.5 \times \mathrm{T}=$ 24.

The correction value is $\mathrm{T} / 4=4$. The signs are evaluated as follows.

$53-56=-3 \rightarrow$ Negative

$27-24=3 \rightarrow$ Positive

$21-24=-3 \rightarrow$ Negative

When these corrections are applied the reconstructed values become

$56-4=52$

$24+4=28$

$24-4=20$

The number of symbols generated is 7 , and three signs. So total 17 bits and in the previous phase 9 bits. Using 26bits it is possible to recover the following.

\begin{tabular}{|c|c|cc|}
\hline 52 & 0 & 28 & 20 \\
\cline { 1 - 2 } 0 & 0 & 0 & 0 \\
\hline 0 & 0 & 0 & 0 \\
0 & 0 & 0 & 0 \\
\hline
\end{tabular}

In the next phase, the threshold becomes 8 . The process will be continued as mentioned above. The symbols generated in each phase along with threshold and number of symbols is given in Table 1.

Table 1. Symbols generated at the end of each phase with Threshold values

\begin{tabular}{|c|c|c|}
\hline Threshold & Sequence of Symbols & $\begin{array}{c}\text { Number of } \\
\text { Symbols }\end{array}$ \\
\hline 32 & 'p- t- t- t' & 04 \\
\hline 16 & 'z- t- t- p- p- t- t' & 07 \\
\hline 8 & $\begin{array}{c}\text { 'z- p- n- p- t- t- p- p- p- n- t- } \\
\text { t- p- t- t- t' }\end{array}$ & 16 \\
\hline 4 & 'z- t- z- z- t- t- p- n- t- n- n- \\
\hline 2 & 'z- t- t- t' & 12 \\
\hline 1 & 'z- t- t- t' & 04 \\
\hline
\end{tabular}

\section{b. SPIHT Coding}

In 1996, another trademark algorithm for image compression based wavelet transform was proposed by A Said and W Pearlman [15]. This is achieved by a direct modification of EZW algorithm. SPIHT makes use of a scheme of coding wavelet coefficients. SPIHT also follows the ordered coding of wavelet coefficients based on the importance of the coefficients and also exploits the selfsimilarity property of subbands. The SPIHT coding also groups the wavelet coefficients in to subsets of insignificant coefficients and identifies the significant ones. Though the SPIHT coding can be thought of as an extension of EZW, because of its success and features of it, it became a state-ofart image compression standard. EZW coding makes use of symbols to denote the type of coefficients that was traced in a traversal. The SPIHT coding uses a set of lists to perform the similar task. For example, consider the following wavelet decomposition.

\begin{tabular}{|c|c|cc|}
\hline 53 & 13 & 27 & 21 \\
\cline { 1 - 2 }-15 & 15 & 13 & 9 \\
\hline 9 & -9 & 9 & -7 \\
5 & -5 & -5 & 0 \\
\hline
\end{tabular}

The SPIHT coding is concern with maintaining the coefficients of wavelet domain in different lists. These are List of insignificant pixels (LIP), list of significant pixels (LSP) and List of insignificant sets (LIS). The SPIHT coding has two passes, sorting and refinement. Three passes at the encoder are presented below and generated the respective transmitted bitstream, then decode this bitstream.

First Pass: The value of $\mathrm{n}$ in this case is 5 and threshold is 32 . The three lists at the encoder are

LIP: $\{(0,0)->53,(0,1)->13,(1,0)->-15,(1,1)->15\}$

LIS: $\{(0,1) \mathrm{D},(1,0) \mathrm{D},(1,1) \mathrm{D}\}$

LSP: \{\}

Beginning the algorithm, we examine the contents of LIP. The coefficient at location $(0,0)$ is greater than 32 . Hence, it is significant. Therefore, a 1 will be transmitted, then a 0 to indicate the coefficient is positive and move the coordinate to LSP. All the remaining coefficients are all insignificant therefore, one 0 will be transmitted for each coefficient and leave these coefficients in LIP. The next step is to examine the contents of LIS. Looking at the descendants of the coefficient at location $(0,1)(27,21,13$ and 9), it can be easily said that none of them are significant, hence a 0 will be transmitted. Similarly, the descendants of the coefficient at location $(1,0)$ and $(1,1)$ are also insignificant. Hence, a 0 will be transmitted for each set. Then, there are no elements in the LSP, hence no refinement at this pass. So, far the bit sequence sent is ' 1000000000 '. The lists at the end of the first pass are given below.

LIP: $\{(0,1)->13,(1,0)->-15,(1,1)->15\}$

LIS: $\{(0,1) \mathrm{D},(1,0) \mathrm{D},(1,1) \mathrm{D}\}$

LSP: $\{(0,0) \rightarrow 53$,

\section{LL BAND CODING SCHEME}

In the standard wavelet based image compression schemes, the input image decomposed using one of the wavelet transforms to obtained wavelet domain of the image. As, already shown in the previous section, the 
wavelet domain contains four bands. In wavelet transformation, one of these bands may be further decomposed depending on the level with which decomposition is being done. The result of decomposition, i.e. the wavelet domain will be coded using different wavelet based compression techniques. If the image is say $256 \times 256$ image, the wavelet decomposition will have $256 \times 256$ elements. These elements will be divided into four decomposition bands of each $128 \times 128$ size. The decomposition bands will have district features, and hence participates in reconstruction in different way. The Low-low (LL) band is the one which carries most of the information and hence influence the reconstruction strongly. If this band is corrupted, the quality of the reconstructed image will be lost. Also, the coefficients in this band will have higher values compared to that in other bands. The wavelet based image compression schemes, as mentioned in the previous section, code these coefficients by comparing with a threshold in each phase, and generates a sequence of symbols in case of EZW and places the indices of coefficients in different lists in case of SPIHT.

The High-high $(\mathrm{HH})$ band, on the other hand, will have coefficients with less value. The coding of these coefficients will lead to more phases, because these values are very less and will be treated as insignificant for many phases. The threshold, which will be used to compare the coefficients to decide whether it is significant or insignificant, will be halved in each phase. Hence, if the coefficient value is very less, then many phases will be required to code all the coefficients. But, when all these coefficients are coded, the perfect reconstruction of original image is possible. For instance, if the smaller coefficients are ignored, i.e. rounded to zero, one can expect that there will be no further changes in last phases. This will reduce the number of symbols generated and lead to less requirement of memory to hold these symbols. This will raise the compression ratio at the cost of quality that might be uphold when these coefficients are not ignored. It is interesting to note that the quality that will be reduced when these coefficients are ignored is very less, and in some cases, there will be no decrease in quality.

But, a question arises on how to categorize or identify the coefficients which are small in value. The observation of wavelet domain or wavelet decomposition will let us know that this categorization can be easily done using the decomposition bands themselves. As mentioned earlier, the LL band has coefficients with higher values, and hence can't be chosen for ignorance. $\mathrm{HH}$ band is the candidate for this section. High-low (HL) and Low-high (LH) bands may also be chosen to increase the coding rate. This is exemplified on both EZW and SPIHT in the following sub-sections.

\section{a. Effect of LL Band Coding on EZW}

For instance, if $\mathrm{HH}$ band is ignored, the decomposition of previous section becomes the following.

\begin{tabular}{|c|c|cc|}
\hline 53 & 13 & 27 & 21 \\
\cline { 1 - 2 }-15 & 15 & 13 & 9 \\
\hline 9 & -9 & 0 & 0 \\
5 & -5 & 0 & 0 \\
\hline
\end{tabular}

The highest number ignored is 9 . Hence, till the threshold is less than 8, the process is as per the regular EZW. But when the threshold drops below 9, then all the coefficients becomes zerotree roots, leading to less number of symbols generated. The respective sequence of symbols and number of symbols are shown in Table 2.

Table 2. Symbols generated at the end of each phase when $\mathrm{HH}$ is ignored

\begin{tabular}{|c|c|c|}
\hline Threshold & \multicolumn{1}{|c|}{ Sequence of Symbols } & $\begin{array}{c}\text { Number of } \\
\text { Symbols }\end{array}$ \\
\hline 32 & 'p- t- t- t' & 04 \\
\hline 16 & 'z- t- t- p- p- t- t' & 07 \\
\hline 8 & $\begin{array}{l}\text { 'z- p- n- p- t- t- p- p- p- n- t- t- t- } \\
\text { t- t- t' }\end{array}$ & 16 \\
\hline 4 & 'z- t- z- t- t- t- p- n' & 08 \\
\hline 2 & 'z- t- t- t' & 04 \\
\hline 1 & 'z- t- t- t' & 04 \\
\hline
\end{tabular}

If the bands $\mathrm{HH}, \mathrm{Hl}$ and $\mathrm{LH}$ are ignored, the symbols generated will be very less and high rate compression is possible. The respective sequence of symbols and number of symbols are shown in Table 3

Table 3. Symbols generated at the end of each phase when HH, LH and HL are ignored

\begin{tabular}{|c|c|c|}
\hline Threshold & \multicolumn{1}{|c|}{ Sequence of Symbols } & $\begin{array}{c}\text { Number of } \\
\text { Symbols }\end{array}$ \\
\hline 32 & 'p- t- t- t' & 04 \\
\hline 16 & 'z- t- t- t' & 04 \\
\hline 8 & $\begin{array}{l}\text { 'z- } \mathrm{p}-\mathrm{n}-\mathrm{p} \text { - t- t- t- t- t- t- t- t- t- } \\
\mathrm{t}-\mathrm{t}-\mathrm{t} \text { ' }\end{array}$ & 16 \\
\hline 4 & 'z- t- t- t' & 04 \\
\hline 2 & 'z- t- t- t' & 04 \\
\hline 1 & 'z- t- t- t' & 04 \\
\hline
\end{tabular}

The tables 2 and 3 show the reduction in length of symbols generated for $4 \times 4$ image. When the size is, say, $256 \times 256$, the effect of selective wavelet band coding will be prevalent.

\section{b. Effect of LL Band Coding on SPIHT}

Insignificant coefficients requires half the number of bits as required by significant bits at level 1 . Insignificant coefficients requires one fourth of the number of bits as required by significant bits at level 2 and so on. A wavelet decomposition of more insignificant coefficients will lead to higher compression rate. A pixel is interpreted as significant or insignificant in different passes by comparing threshold in that pass.

\section{SIMULATION RESULTS}

In this section, the compression results of the techniques presented in the above sections are presented. All standard wavelets were considered and simulation was carried out on many test case images and results on few images are presented in this section. Interestingly in EZW case, even 
when few bands are ignored completely the PSNR was increased slightly. This is because the coefficients ignored carries no information and in addition, they create some ambiguity in the decompression. When these coefficients were ignored, the PSNR was raised slightly. The test images considered in this work are shown in Figure 2.
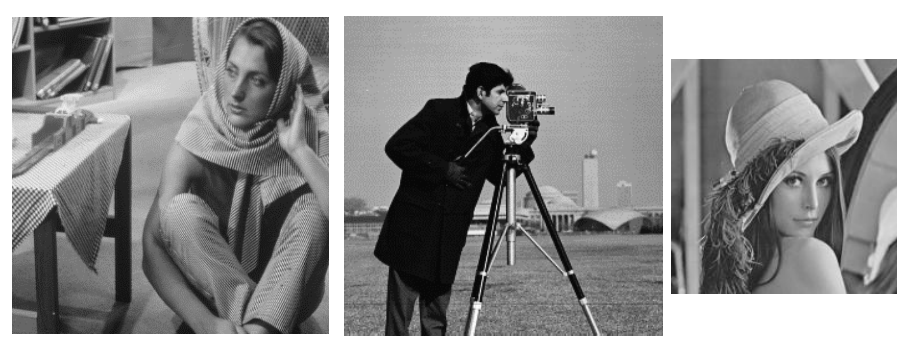

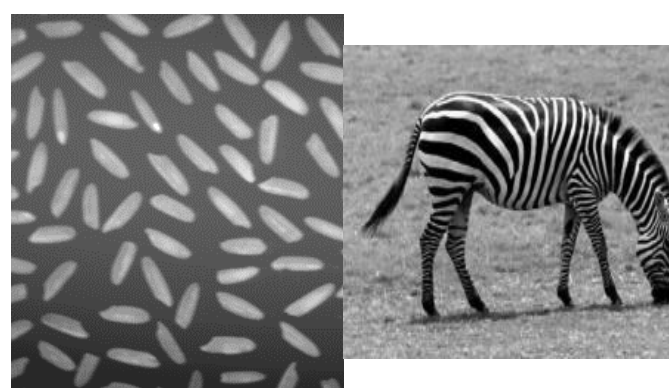

Fig. 2. Test images

The compression results obtained with EZW are given in Tables 4 to 11 for different test images.
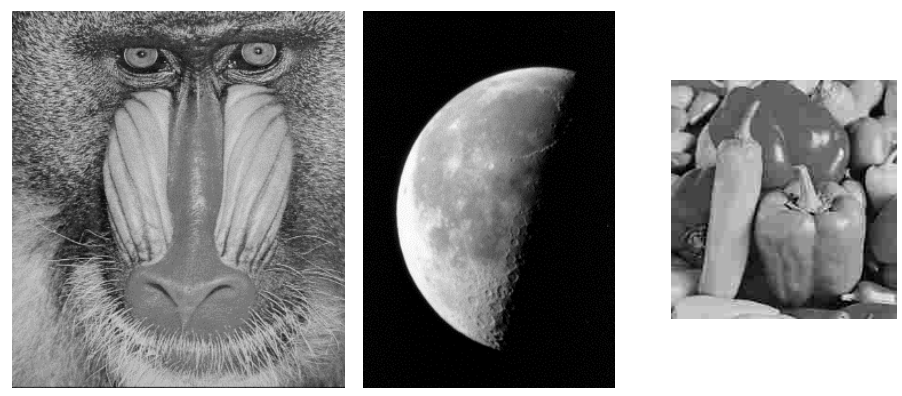

Table 4. Compression results using EZW and modified EZW on 'barbara' image

\begin{tabular}{|c|c|c|c|c|c|c|c|c|c|}
\hline \multirow[b]{2}{*}{ Wavelet } & \multicolumn{3}{|c|}{ Normal } & \multicolumn{3}{|c|}{$\mathrm{HH}=0$} & \multicolumn{3}{|c|}{$\mathrm{HH}=\mathrm{HL}=\mathrm{LH}=0$} \\
\hline & $\begin{array}{c}\text { PSNR } \\
(\mathrm{dB})\end{array}$ & CR & SSIM & $\begin{array}{c}\text { PSNR } \\
(\mathrm{dB})\end{array}$ & CR & SSIM & $\begin{array}{c}\text { PSNR } \\
(\mathrm{dB})\end{array}$ & CR & SSIM \\
\hline Haar & 24.95 & 2.61 & 0.47 & 24.95 & 2.61 & 0.47 & 25.05 & 2.71 & 0.47 \\
\hline DB & 26.02 & 2.97 & 0.54 & 26.02 & 2.97 & 0.54 & 26.16 & 3.05 & 0.54 \\
\hline Bior & 25.03 & 2.22 & 0.49 & 25.03 & 2.22 & 0.49 & 25.13 & 2.32 & 0.49 \\
\hline Coif & 26.35 & 3.11 & 0.55 & 26.35 & 3.11 & 0.55 & 26.46 & 3.17 & 0.55 \\
\hline Sym & 25.80 & 2.72 & 0.53 & 25.80 & 2.72 & 0.53 & 25.91 & 2.77 & 0.53 \\
\hline Dmey & 26.38 & 3.04 & 0.54 & 26.38 & 3.04 & 0.54 & 26.45 & 3.08 & 0.55 \\
\hline
\end{tabular}

Table 5. Compression results using EZW and modified EZW on 'cameraman' image

\begin{tabular}{|l|l|l|l|l|l|l|l|l|l|}
\hline \multirow{2}{*}{ Wavelet } & \multicolumn{3}{|c|}{ Normal } & \multicolumn{3}{c|}{ HH=0 } & \multicolumn{3}{c|}{ HH=HL=LH=0 } \\
\cline { 2 - 11 } & PSNR & CR & SSIM & PSNR & CR & SSIM & PSNR & CR & SSIM \\
\hline Haar & 24.41 & 2.65 & 0.27 & 24.43 & 2.68 & 0.27 & 24.29 & 3.35 & 0.26 \\
\hline DB & 24.63 & 2.65 & 0.28 & 24.65 & 2.68 & 0.28 & 24.92 & 3.03 & 0.29 \\
\hline Bior & 24.42 & 2.37 & 0.27 & 24.45 & 2.39 & 0.27 & 24.25 & 2.92 & 0.27 \\
\hline Coif & 24.87 & 2.76 & 0.28 & 24.88 & 2.80 & 0.28 & 25.11 & 3.21 & 0.29 \\
\hline Sym & 23.66 & 2.60 & 0.26 & 23.68 & 2.63 & 0.26 & 24.63 & 3.07 & 0.28 \\
\hline Dmey & 24.58 & 2.71 & 0.27 & 24.61 & 2.74 & 0.27 & 25.14 & 3.03 & 0.28 \\
\hline
\end{tabular}

Table 6. Compression results using EZW and modified EZW on 'lena' image

\begin{tabular}{|l|l|l|l|l|l|l|l|c|c|}
\hline \multirow{2}{*}{ Wavelet } & \multicolumn{3}{|c|}{ Normal } & \multicolumn{3}{c|}{ HH=0 } & \multicolumn{3}{c|}{ HH=HL=LH=0 } \\
\cline { 2 - 11 } & PSNR & CR & SSIM & PSNR & CR & SSIM & PSNR & CR & SSIM \\
\hline Haar & 27.32 & 4.10 & 0.37 & 27.32 & 4.10 & 0.37 & 27.30 & 4.22 & 0.37 \\
\hline DB & 28.17 & 4.53 & 0.45 & 28.17 & 4.53 & 0.45 & 28.24 & 4.59 & 0.45 \\
\hline Bior & 27.39 & 3.39 & 0.39 & 27.39 & 3.39 & 0.39 & 27.40 & 3.49 & 0.39 \\
\hline Coif & 28.40 & 4.71 & 0.47 & 28.40 & 4.71 & 0.47 & 28.47 & 4.75 & 0.47 \\
\hline Sym & 27.80 & 4.13 & 0.43 & 27.80 & 4.13 & 0.43 & 27.90 & 4.18 & 0.44 \\
\hline Dmey & 28.43 & 4.53 & 0.46 & 28.43 & 4.53 & 0.46 & 28.47 & 4.55 & 0.47 \\
\hline
\end{tabular}

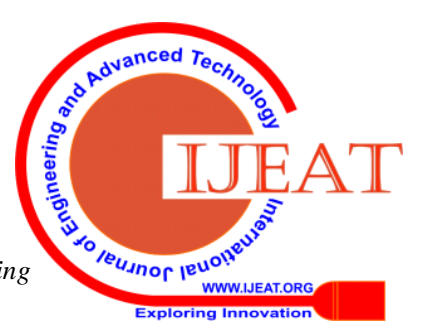


HIGH RATE SQUEEZING USING LL BAND CODING IN WAVELET DOMAIN

Table 7. Compression results using EZW and modified EZW on 'mandril' image

\begin{tabular}{|l|c|c|c|c|c|c|c|c|c|}
\hline \multirow{2}{*}{ Wavelet } & \multicolumn{3}{|c|}{ Normal } & \multicolumn{3}{c|}{ HH=0 } & \multicolumn{3}{c|}{ HH=HL=LH=0 } \\
\cline { 2 - 10 } & PSNR & CR & SSIM & PSNR & CR & SSIM & PSNR & CR & SSIM \\
\hline Haar & 23.00 & 3.04 & 0.33 & 23.00 & 3.05 & 0.33 & 23.00 & 3.33 & 0.32 \\
\hline DB & 23.50 & 3.21 & 0.40 & 23.50 & 3.21 & 0.40 & 23.49 & 3.55 & 0.39 \\
\hline Bior & 23.08 & 2.51 & 0.37 & 23.08 & 2.51 & 0.37 & 23.08 & 2.72 & 0.36 \\
\hline Coif & 23.53 & 3.20 & 0.40 & 23.53 & 3.21 & 0.40 & 23.56 & 3.57 & 0.39 \\
\hline Sym & 23.28 & 3.22 & 0.37 & 23.28 & 3.22 & 0.37 & 23.31 & 3.53 & 0.36 \\
\hline Dmey & 23.61 & 3.21 & 0.42 & 23.61 & 3.21 & 0.42 & 23.62 & 3.50 & 0.41 \\
\hline
\end{tabular}

Table 8. Compression results using EZW and modified EZW on 'moon' image

\begin{tabular}{|l|c|c|c|c|c|c|c|c|c|}
\hline \multirow{2}{*}{ Wavelet } & \multicolumn{4}{|c|}{ Normal } & \multicolumn{3}{c|}{ HH=0 } & \multicolumn{3}{c|}{ HH=HL=LH=0 } \\
\cline { 2 - 10 } & PSNR & CR & SSIM & PSNR & CR & SSIM & PSNR & CR & SSIM \\
\hline Haar & 31.25 & 6.03 & 0.17 & 31.25 & 6.03 & 0.17 & 29.76 & 6.74 & 0.16 \\
\hline DB & 31.26 & 7.41 & 0.19 & 31.26 & 7.41 & 0.19 & 31.26 & 7.42 & 0.19 \\
\hline Bior & 31.31 & 5.23 & 0.17 & 31.31 & 5.23 & 0.17 & 29.73 & 5.79 & 0.17 \\
\hline Coif & 31.59 & 7.85 & 0.19 & 31.59 & 7.85 & 0.19 & 31.58 & 7.86 & 0.19 \\
\hline Sym & 31.46 & 7.26 & 0.19 & 31.46 & 7.26 & 0.19 & 31.26 & 7.43 & 0.19 \\
\hline Dmey & 31.39 & 7.21 & 0.20 & 31.39 & 7.21 & 0.20 & 31.39 & 7.21 & 0.20 \\
\hline
\end{tabular}

Table 9. Compression results using EZW and modified EZW on 'pepper' image

\begin{tabular}{|l|c|c|c|c|c|c|c|c|c|}
\hline \multirow{2}{*}{ Wavelet } & \multicolumn{3}{|c|}{ Normal } & \multicolumn{3}{c|}{ HH=0 } & \multicolumn{3}{c|}{ HH=HL=LH=0 } \\
\cline { 2 - 10 } & PSNR & CR & SSIM & PSNR & CR & SSIM & PSNR & CR & SSIM \\
\hline Haar & 26.23 & 2.81 & 0.47 & 26.23 & 2.81 & 0.47 & 26.32 & 2.92 & 0.47 \\
\hline DB & 27.52 & 3.13 & 0.54 & 27.52 & 3.13 & 0.54 & 27.72 & 3.21 & 0.55 \\
\hline Bior & 26.24 & 2.44 & 0.49 & 26.24 & 2.44 & 0.49 & 26.38 & 2.53 & 0.49 \\
\hline Coif & 27.88 & 3.32 & 0.54 & 27.88 & 3.32 & 0.54 & 28.01 & 3.37 & 0.55 \\
\hline Sym & 27.05 & 2.95 & 0.54 & 27.05 & 2.95 & 0.54 & 27.37 & 3.02 & 0.55 \\
\hline Dmey & 27.94 & 3.19 & 0.55 & 27.94 & 3.19 & 0.55 & 28.03 & 3.22 & 0.55 \\
\hline
\end{tabular}

Table 10. Compression results using EZW and modified EZW on 'rice' image

\begin{tabular}{|l|c|c|c|c|c|c|c|c|c|}
\hline \multirow{2}{*}{ Wavelet } & \multicolumn{3}{|c|}{ Normal } & \multicolumn{3}{c|}{ HH=0 } & \multicolumn{3}{c|}{ HH=HL=LH=0 } \\
\cline { 2 - 10 } & PSNR & CR & SSIM & PSNR & CR & SSIM & PSNR & CR & SSIM \\
\hline Haar & 27.07 & 2.27 & 0.57 & 27.07 & 2.27 & 0.57 & 27.07 & 2.27 & 0.57 \\
\hline DB & 28.39 & 3.51 & 0.59 & 28.39 & 3.51 & 0.59 & 28.41 & 3.54 & 0.59 \\
\hline Bior & 27.25 & 1.96 & 0.58 & 27.25 & 1.96 & 0.58 & 27.25 & 1.96 & 0.58 \\
\hline Coif & 28.84 & 3.40 & 0.60 & 28.84 & 3.40 & 0.60 & 28.87 & 3.41 & 0.60 \\
\hline Sym & 27.78 & 2.84 & 0.57 & 27.78 & 2.84 & 0.57 & 27.86 & 2.85 & 0.57 \\
\hline Dmey & 28.89 & 3.61 & 0.60 & 28.89 & 3.61 & 0.60 & 28.90 & 3.62 & 0.60 \\
\hline
\end{tabular}

Table 11. Compression results using EZW and modified EZW on 'zebra' image

\begin{tabular}{|l|c|c|c|c|c|c|c|c|c|}
\hline \multirow{2}{*}{ Wavelet } & \multicolumn{3}{|c|}{ Normal } & \multicolumn{3}{c|}{ HH=0 } & \multicolumn{3}{c|}{ HH=HL=LH=0 } \\
\cline { 2 - 10 } & PSNR & CR & SSIM & PSNR & CR & SSIM & PSNR & CR & SSIM \\
\hline Haar & 22.54 & 1.79 & 0.42 & 22.54 & 1.79 & 0.42 & 22.72 & 2.14 & 0.42 \\
\hline DB & 25.77 & 2.21 & 0.53 & 25.77 & 2.21 & 0.53 & 25.17 & 2.33 & 0.52 \\
\hline Bior & 21.87 & 1.53 & 0.45 & 21.87 & 1.53 & 0.45 & 22.74 & 1.80 & 0.46 \\
\hline
\end{tabular}




\begin{tabular}{|l|l|l|l|l|l|l|l|l|l|}
\hline Coif & 25.92 & 2.23 & 0.52 & 25.92 & 2.23 & 0.52 & 25.38 & 2.36 & 0.51 \\
\hline Sym & 24.02 & 2.05 & 0.47 & 24.02 & 2.05 & 0.47 & 24.12 & 2.24 & 0.47 \\
\hline Dmey & 25.45 & 2.24 & 0.52 & 25.45 & 2.24 & 0.52 & 25.66 & 2.32 & 0.52 \\
\hline
\end{tabular}

Similarly the compression results using SPIHT and modified SPIHT on different image are given in Tables 12 to 19 .

Table 12. Compression results using SPIHT and modified SPIHT on 'barbara' image

\begin{tabular}{|c|c|c|c|c|c|c|c|c|c|}
\hline \multirow{2}{*}{ Wavelet } & \multicolumn{3}{|c|}{ Normal } & \multicolumn{3}{c|}{ HH=0 } & \multicolumn{3}{c|}{ HH=HL=LH=0 } \\
\cline { 2 - 10 } & PSNR & CR & SSIM & PSNR & CR & SSIM & PSNR & CR & SSIM \\
\hline Haar & 36.82 & 2.36 & 0.97 & 33.77 & 2.64 & 0.94 & 27.01 & 3.82 & 0.83 \\
\hline DB & 37.14 & 2.35 & 0.97 & 34.14 & 2.61 & 0.94 & 29.09 & 3.59 & 0.87 \\
\hline Bior & 30.54 & 2.29 & 0.93 & 29.90 & 2.57 & 0.91 & 26.85 & 3.70 & 0.82 \\
\hline Coif & 37.16 & 2.22 & 0.97 & 34.21 & 2.46 & 0.94 & 29.12 & 3.39 & 0.87 \\
\hline Sym & 29.41 & 2.41 & 0.93 & 28.88 & 2.69 & 0.91 & 26.73 & 3.75 & 0.84 \\
\hline Dmey & 33.26 & 1.30 & 0.95 & 32.28 & 1.44 & 0.93 & 29.35 & 1.98 & 0.87 \\
\hline
\end{tabular}

Table 13. Compression results using SPIHT and modified SPIHT on 'cameraman' image

\begin{tabular}{|c|c|c|c|c|c|c|c|c|c|}
\hline \multirow{2}{*}{ Wavelet } & \multicolumn{3}{|c|}{ Normal } & \multicolumn{3}{c|}{ HH=0 } & \multicolumn{3}{c|}{ HH=HL=LH=0 } \\
\cline { 2 - 10 } & PSNR & CR & SSIM & PSNR & CR & SSIM & PSNR & CR & SSIM \\
\hline Haar & 37.69 & 2.46 & 0.96 & 33.51 & 2.75 & 0.95 & 25.03 & 3.82 & 0.83 \\
\hline DB & 37.38 & 2.19 & 0.96 & 31.27 & 2.43 & 0.91 & 24.69 & 3.25 & 0.73 \\
\hline Bior & 28.31 & 2.24 & 0.93 & 27.26 & 2.49 & 0.88 & 23.69 & 3.35 & 0.70 \\
\hline Coif & 37.40 & 2.07 & 0.96 & 34.38 & 2.28 & 0.95 & 24.76 & 3.06 & 0.73 \\
\hline Sym & 26.39 & 2.30 & 0.92 & 25.73 & 2.54 & 0.88 & 23.06 & 3.39 & 0.70 \\
\hline Dmey & 26.39 & 2.30 & 0.92 & 29.65 & 1.33 & 0.92 & 24.95 & 1.79 & 0.74 \\
\hline
\end{tabular}

Table 14. Compression results using SPIHT and modified SPIHT on 'lena' image

\begin{tabular}{|c|c|c|c|c|c|c|c|c|c|}
\hline \multirow{2}{*}{ Wavelet } & \multicolumn{3}{|c|}{ Normal } & \multicolumn{3}{c|}{ HH=0 } & \multicolumn{3}{c|}{ HH=HL=LH=0 } \\
\cline { 2 - 10 } & PSNR & CR & SSIM & PSNR & CR & SSIM & PSNR & CR & SSIM \\
\hline Haar & 37.91 & 2.58 & 0.97 & 36.67 & 2.81 & 0.96 & 29.60 & 3.82 & 0.88 \\
\hline DB & 38.27 & 2.61 & 0.97 & 37.62 & 2.81 & 0.97 & 31.71 & 3.59 & 0.92 \\
\hline Bior & 33.16 & 2.50 & 0.94 & 32.90 & 2.72 & 0.94 & 29.39 & 3.70 & 0.88 \\
\hline Coif & 38.32 & 2.48 & 0.97 & 37.69 & 2.66 & 0.97 & 31.75 & 3.39 & 0.92 \\
\hline Sym & 30.98 & 2.65 & 0.94 & 30.84 & 2.87 & 0.94 & 28.78 & 3.75 & 0.89 \\
\hline Dmey & 36.22 & 1.47 & 0.96 & 35.99 & 1.57 & 0.96 & 31.95 & 1.98 & 0.92 \\
\hline
\end{tabular}

Table 15. Compression results using SPIHT and modified SPIHT on 'mandril' image

\begin{tabular}{|c|c|c|c|c|c|c|c|c|c|}
\hline \multirow{2}{*}{ Wavelet } & \multicolumn{3}{|c|}{ Normal } & \multicolumn{3}{c|}{ HH=0 } & \multicolumn{3}{c|}{ HH=HL=LH=0 } \\
\cline { 2 - 10 } & PSNR & CR & SSIM & PSNR & CR & SSIM & PSNR & CR & SSIM \\
\hline Haar & 35.83 & 2.14 & 0.97 & 31.77 & 2.47 & 0.94 & 24.90 & 3.82 & 0.73 \\
\hline DB & 35.76 & 2.06 & 0.97 & 32.69 & 2.36 & 0.95 & 25.90 & 3.59 & 0.78 \\
\hline Bior & 28.09 & 2.07 & 0.90 & 27.45 & 2.39 & 0.88 & 24.75 & 3.70 & 0.73 \\
\hline Coif & 35.81 & 1.94 & 0.97 & 32.75 & 2.23 & 0.95 & 25.89 & 3.39 & 0.79 \\
\hline Sym & 27.90 & 2.14 & 0.92 & 27.31 & 2.46 & 0.90 & 24.44 & 3.75 & 0.75 \\
\hline Dmey & 29.72 & 1.13 & 0.92 & 29.12 & 1.29 & 0.91 & 26.02 & 1.98 & 0.79 \\
\hline
\end{tabular}


HIGH RATE SQUEEZING USING LL BAND CODING IN WAVELET DOMAIN

Table 16. Compression results using SPIHT and modified SPIHT on 'moon' image

\begin{tabular}{|c|c|c|c|c|c|c|c|c|c|}
\hline \multirow{2}{*}{ Wavelet } & \multicolumn{3}{|c|}{ Normal } & \multicolumn{3}{c|}{ HH=0 } & \multicolumn{3}{c|}{ HH=HL=LH=0 } \\
\cline { 2 - 10 } & PSNR & CR & SSIM & PSNR & CR & SSIM & PSNR & CR & SSIM \\
\hline Haar & 38.79 & 2.75 & 0.81 & 38.11 & 2.98 & 0.81 & 30.85 & 3.82 & 0.58 \\
\hline DB & 38.84 & 2.71 & 0.81 & 38.63 & 2.90 & 0.81 & 33.43 & 3.59 & 0.60 \\
\hline Bior & 34.08 & 2.50 & 0.79 & 33.94 & 2.70 & 0.79 & 30.72 & 3.36 & 0.58 \\
\hline Coif & 38.86 & 2.38 & 0.82 & 38.65 & 2.53 & 0.81 & 33.53 & 3.07 & 0.60 \\
\hline Sym & 37.53 & 2.82 & 0.81 & 37.35 & 3.02 & 0.80 & 32.85 & 3.76 & 0.59 \\
\hline Dmey & 37.64 & 1.41 & 0.82 & 37.56 & 1.49 & 0.82 & 33.81 & 1.79 & 0.60 \\
\hline
\end{tabular}

Table 17. Compression results using SPIHT and modified SPIHT on 'pepper' image

\begin{tabular}{|c|c|c|c|c|c|c|c|c|c|}
\hline \multirow{2}{*}{ Wavelet } & \multicolumn{3}{|c|}{ Normal } & \multicolumn{3}{c|}{ HH=0 } & \multicolumn{3}{c|}{ HH=HL=LH=0 } \\
\cline { 2 - 10 } & PSNR & CR & SSIM & PSNR & CR & SSIM & PSNR & CR & SSIM \\
\hline Haar & 37.97 & 2.65 & 0.98 & 37.26 & 2.83 & 0.97 & 28.68 & 3.82 & 0.90 \\
\hline DB & 38.38 & 2.72 & 0.98 & 38.26 & 2.81 & 0.98 & 31.93 & 3.59 & 0.94 \\
\hline Bior & 31.86 & 2.53 & 0.94 & 31.75 & 2.73 & 0.94 & 28.47 & 3.70 & 0.90 \\
\hline Coif & 38.50 & 2.58 & 0.98 & 38.33 & 2.66 & 0.98 & 31.95 & 3.39 & 0.94 \\
\hline Sym & 29.34 & 2.78 & 0.95 & 29.32 & 2.93 & 0.95 & 27.87 & 3.75 & 0.91 \\
\hline Dmey & 35.93 & 1.47 & 0.97 & 35.88 & 1.53 & 0.97 & 32.38 & 1.98 & 0.94 \\
\hline
\end{tabular}

Table 18. Compression results using SPIHT and modified SPIHT on 'rice' image

\begin{tabular}{|c|c|c|c|c|c|c|c|c|c|}
\hline \multirow{2}{*}{ Wavelet } & \multicolumn{3}{|c|}{ Normal } & \multicolumn{3}{c|}{ HH=0 } & \multicolumn{3}{c|}{ HH=HL=LH=0 } \\
\cline { 2 - 10 } & PSNR & CR & SSIM & PSNR & CR & SSIM & PSNR & CR & SSIM \\
\hline Haar & 38.33 & 2.71 & 0.98 & 38.25 & 2.91 & 0.98 & 30.04 & 3.82 & 0.93 \\
\hline DB & 38.96 & 2.57 & 0.98 & 34.05 & 2.70 & 0.97 & 28.51 & 3.25 & 0.93 \\
\hline Bior & 34.02 & 2.45 & 0.96 & 31.64 & 2.62 & 0.94 & 26.88 & 3.35 & 0.89 \\
\hline Coif & 39.08 & 2.43 & 0.99 & 34.11 & 2.56 & 0.97 & 28.57 & 3.06 & 0.93 \\
\hline Sym & 29.13 & 2.61 & 0.96 & 28.38 & 2.75 & 0.94 & 26.36 & 3.39 & 0.90 \\
\hline Dmey & 39.08 & 1.44 & 0.98 & 34.28 & 1.50 & 0.97 & 28.63 & 1.79 & 0.93 \\
\hline
\end{tabular}

Table 19. Compression results using SPIHT and modified SPIHT on 'rice' image

\begin{tabular}{|c|c|c|c|c|c|c|c|c|c|}
\hline \multirow{2}{*}{ Wavelet } & \multicolumn{4}{|c|}{ Normal } & \multicolumn{3}{c|}{ HH=0 } & \multicolumn{3}{c|}{ HH=HL=LH=0 } \\
\cline { 2 - 10 } & PSNR & CR & SSIM & PSNR & CR & SSIM & PSNR & CR & SSIM \\
\hline Haar & 36.34 & 2.25 & 0.97 & 34.65 & 2.52 & 0.96 & 24.12 & 3.82 & 0.85 \\
\hline DB & 37.14 & 2.23 & 0.98 & 36.89 & 2.36 & 0.98 & 25.83 & 3.25 & 0.88 \\
\hline Bior & 27.50 & 2.06 & 0.93 & 27.34 & 2.28 & 0.93 & 23.09 & 3.36 & 0.81 \\
\hline Coif & 37.21 & 2.13 & 0.98 & 37.00 & 2.24 & 0.98 & 26.05 & 3.07 & 0.88 \\
\hline Sym & 24.67 & 2.21 & 0.93 & 24.63 & 2.39 & 0.93 & 22.37 & 3.39 & 0.83 \\
\hline Dmey & 32.79 & 1.29 & 0.97 & 32.76 & 1.34 & 0.97 & 26.51 & 1.79 & 0.89 \\
\hline
\end{tabular}

The average values of PSNR, Compression ratio and structural similarity for different test image with each wavelet is given Tables 20 and 21 . 
International Journal of Engineering and Advanced Technology (IJEAT) ISSN: 2249 - 8958, Volume-8 Issue-6S, August 2019

Table 20. Average values of PSNR, CR and SSIM of different test images with EZW and modified EZW

\begin{tabular}{|l|c|c|c|c|c|c|c|c|c|}
\hline \multirow{2}{*}{ Wavelet } & \multicolumn{3}{|c|}{ Normal } & \multicolumn{3}{c|}{ HH=0 } & \multicolumn{3}{c|}{ HH=HL=LH=0 } \\
\cline { 2 - 10 } & PSNR & CR & SSIM & PSNR & CR & SSIM & PSNR & CR & SSIM \\
\hline Haar & 25.85 & 3.16 & 0.38 & 25.85 & 3.17 & 0.38 & 25.69 & 3.46 & 0.38 \\
\hline DB & 26.91 & 3.70 & 0.44 & 26.91 & 3.71 & 0.44 & 26.92 & 3.84 & 0.44 \\
\hline Bior & 25.82 & 2.71 & 0.40 & 25.83 & 2.71 & 0.40 & 25.75 & 2.94 & 0.40 \\
\hline Coif & 27.17 & 3.82 & 0.44 & 27.17 & 3.83 & 0.44 & 27.18 & 3.96 & 0.44 \\
\hline Sym & 26.36 & 3.47 & 0.42 & 26.36 & 3.47 & 0.42 & 26.54 & 3.64 & 0.42 \\
\hline Dmey & 27.08 & 3.72 & 0.45 & 27.09 & 3.72 & 0.45 & 27.21 & 3.81 & 0.45 \\
\hline
\end{tabular}

Table 21. Average values of PSNR, CR and SSIM of different test images with SPIHT and modified SPIHT

\begin{tabular}{|l|c|c|c|c|c|c|c|c|c|}
\hline \multirow{2}{*}{ Wavelet } & \multicolumn{3}{|c|}{ Normal } & \multicolumn{3}{c|}{ HH=0 } & \multicolumn{3}{c|}{ HH=HL=LH=0 } \\
\cline { 2 - 10 } & PSNR & CR & SSIM & PSNR & CR & SSIM & PSNR & CR & SSIM \\
\hline Haar & 37.46 & 2.49 & 0.95 & 35.50 & 2.74 & 0.94 & 27.53 & 3.82 & 0.82 \\
\hline DB & 37.73 & 2.43 & 0.95 & 35.44 & 2.62 & 0.94 & 28.89 & 3.47 & 0.83 \\
\hline Bior & 30.94 & 2.33 & 0.91 & 30.27 & 2.56 & 0.90 & 26.73 & 3.53 & 0.79 \\
\hline Coif & 37.79 & 2.28 & 0.95 & 35.89 & 2.45 & 0.94 & 28.95 & 3.22 & 0.83 \\
\hline Sym & 29.42 & 2.49 & 0.92 & 29.05 & 2.70 & 0.91 & 26.56 & 3.62 & 0.80 \\
\hline Dmey & 33.88 & 1.47 & 0.94 & 33.44 & 1.44 & 0.93 & 29.20 & 1.88 & 0.83 \\
\hline
\end{tabular}

Table 22 gives complete summary of the proposed techniques in this paper. The average performance by considering different wavelets and different test images is given in Table 22.

Table 22. Summary of Compression results using existing and proposed methods

\begin{tabular}{|l|c|c|c|c|c|c|c|c|c|}
\hline \multirow{2}{*}{ Coding Technique } & \multicolumn{3}{|c|}{ Normal } & \multicolumn{3}{c|}{ HH=0 } & \multicolumn{3}{c|}{ HH=HL=LH=0 } \\
\cline { 2 - 11 } & PSNR & CR & SSIM & PSNR & CR & SSIM & PSNR & CR & SSIM \\
\hline EZW & 26.53 & 3.43 & 0.42 & 26.53 & 3.43 & 0.42 & 26.55 & 3.61 & 0.42 \\
\hline SPIHT & 34.54 & 2.25 & 0.94 & 33.27 & 2.42 & 0.93 & 27.98 & 3.26 & 0.82 \\
\hline
\end{tabular}

From Table 22, it is clear that there is a little improvement in PSNR and a significant improvement of compression ratio when EZW is used. With SPIHT, a decrement in PSNR and substantial improvement in compression ratio is observed.

\section{CONCLUSIONS}

In this paper, an attempt has been made to propose a modification to wavelet domain, such that the compression ratio in image compression is improved. The intention is to improve the compression at the cost of some quality in the reconstructed image. But, in many cases, the improvement in compression ratio did not cost any quality. Indeed, the quality was also raised. This happened with EZW coding. When SPIHT coding is employed, for $\mathrm{HH}=0$, the changes to both compression ratio and quality of the reconstructed image is nominal. But, when only LL band is coded by ignoring the remaining bands, the PSNR was dropped by about $20 \%$ while the improvement in compression ratio is about $45 \%$. Hence, the proposed scheme can be employed in allocations where compression ratio is the main criteria.

\section{REFERENCES}

1. I. JTC1/SC29/WG1, "JPEG 2000 - lossless and lossy compression of continuous- tone and bi-level still images",
Part 1: Minimum decoder. Final committee draft, Version 1.0., March 2000.

2. G.Wallace, "The JPEG still picture compression standard", IEEE TCE, 38, 1992.

3. Independent JPEG Group, version 6a:http://www.ijg.org.

4. G. K. Wallace, "The JPEG still picture compression standard", in IEEE Transactions on Circuits and Systems for Video Technology, vol. 6, June 1996.

5. O. K. Al-Shaykh, "JPEG-2000: A new still image compression standard", in Conference Record of ThirtySecond Asilomar Conference on Signals Systems and Computers, vol. 1, pp. 99-103, 1998.

6. JPEG2000, http://www.jpeg.org/JPEG2000.htm.

7. Moving Picture Expert Group Standard, http://www.mpeg. org/MPEG/index.html.

8. S.Mallat, "Multifrequency channel decompositions of images and wavelet models", IEEE Trans. Acous. Speech Sig. Proc., vol. 37, 12, pp. 2091-2110, 1989.

9. I.Daubechies, "Ten Lectures on Wavelets", SIAM, 1992.

10. E.P.Simoncelli et al., "Shiftable multiscale transforms", IEEE Trans. Information Th., vol.38, 2, pp. 587-607, 1992.

11. Shapiro J.M., "Embedded image coding using zerotrees of wavelet coefficients", IEEE Trans. Signal Proc., Vol. 41, No. 12, pp. 3445-3462, 1993. 
12. Jaya Krishna Sunkara, E Navaneethasagari, D Pradeep, E Naga Chaithanya, D Pavani, D V Sai Sudheer, "A New Video Compression Method using DCT/DWT and SPIHT based on Accordion Representation", I.J. Image, Graphics and Signal Processing, pp. 28-34, May 2012.

13. Jaya Krishna Sunkara, Purnima Kuruma, Ravi Sankaraiah Y, "Image Compression Using Hand Designed and Lifting Based Wavelet Transforms", International Journal of Electronics Communications and Computer Technology, vol. 2 (4), 2012.

14. Jaya Krishna Sunkara, K Subramanyam, V. V. Satyanarayana Tallapragada, V Kiran Kumar, "Image Compression by SPIHT Coding using Traditional Wavelets," Journal of Emerging Technologies and Innovative Research (JETIR), vol. 5, issue 5, pp. 256-267, May 2018.

15. Amir Said, and Pearlman, W. A. A New, Fast, and Efficient Image Codec Based on Set Partitioning in Hierarchical Trees, IEEE Trans. Circuit and systems for Video Technology, vol. 6, no. 3, June 1996, pp.243-250. 